\title{
Influence Characteristics of Laser Transmission Amplitude Fluctuation Based on Turbulent Medium
}

\author{
Xiaodong Yang, Ming Gao \\ Xi'an Technological University, Xi'an, China \\ Email:kevin09409@sina.com,minggao1964@163.com
}

How to cite this paper: Yang, X.D. and Gao, M. (2020) Influence Characteristics of Laser Transmission Amplitude Fluctuation Based on Turbulent Medium. Optics and Photonics Journal, 10, 95-104. https://doi.org/10.4236/opj.2020.106009

Received: March 13, 2020

Accepted: June 26, 2020

Published: June 29, 2020

\begin{abstract}
In order to study the transmission characteristics of laser in atmospheric turbulent medium and understand the influence degree of various factors on amplitude fluctuation, by means of smooth perturbation method, this paper establishes a theory model of amplitude fluctuation of laser propagation in turbulent medium by using the smooth perturbation method and reflects the amplitude fluctuation degree, carries out specific discussion on each influence factor. The results show that the larger the wavelength, the more stable the amplitude fluctuation. With the increase of laser section radius, the amplitude fluctuates sharply and then decreases slowly after reaching the peak. Transmission distance is the main influence factor of amplitude fluctuation. With the increase of transmission distance, the amplitude fluctuation will become more obvious. The amplitude acquisition can be comprehensively modulated in a specific transmission distance by wavelength and section radius, so as to ensure the stability of the received laser and provide a theoretical basis for the interferometry technology.
\end{abstract}

\section{Keywords}

Amplitude Fluctuation, Turbulent Medium, Smooth Perturbation Method, Section Radius, Interferometry

\section{Introduction}

It is an important method in communication [1] and detection [2] to use the optical wave as carrier to transmit information. If there is no influence of atmospheric turbulence, the echo signal of detected target [3] [4] remains unchanged. The value of echo signal is determined by transmission shape of 
transmitter and diffraction effect, which is easy to be observed. However, there is atmospheric turbulence in reality. It makes the atmospheric attributes change random and continuously [5] [6] [7], thus it influences the received signal inevitably. The change of optical wave attributes caused by medium change should be taken into consideration so as to detect the target accurately. The noise generated by atmosphere [8] [9] [10] can reduce the reception performance of system and can disturb the accuracy of acquired signal. The reception perturbation of signal [11] [12] is more obvious in remote sensing application, thus it should be studied deeply using related theory.

The amplitude is an important attribute of optical wave, which not only includes the intensity information, but also has obvious influence on interference technology [13] [14] [15]. The fluctuation rule of amplitude is an important indicator of propagation of light laser, which can reflect the random magnitude of turbulence fluctuation objectively and can provide beneficial support for processing the received signal. The interference radar has been paid much attention as it has high resolution, high accuracy and can work in all weather. To make the interference information more precise, the amplitude fluctuation rule of light laser propagating in the turbulence medium should be studied, the change values and parameters of amplitude should be analyzed quantitatively, thus the reference laser can be compensated, so as to assure there is interference. This plays an important role in military, transportation, agriculture and manufacture.

To solve the transmission and interference issue of laser in atmospheric turbulence medium, this paper established the theoretical model of amplitude fluctuation by using the concepts in statistics and the smooth perturbation method [16] [17] from the perspective of medium fluctuation of atmospheric turbulence. This paper is of practical significance for studying the interference measurement technology and can provide theoretical basis for interference radar detecting target detection in long distance.

\section{Method}

The scalar Helmholtz equation [18] is introduced into the medium fluctuation caused by atmospheric turbulence, thus

$$
\nabla^{2} E+k^{2}[1+\tilde{\varepsilon}(\varrho, z)] E=0
$$

where $E$ is the wave function; $k=\frac{2 \pi}{\lambda}=\frac{\omega}{v}$ is the wave number; and $\tilde{\varepsilon}(\varrho, z)$ is the relative fluctuation of dielectric constant. The wave function of transmission laser is defined as

$$
\tilde{E}(\varrho, z)=A_{0} \exp (i k z)
$$

where $A_{0}$ is the transmission amplitude of optical source. The following formula can be obtained by substituting Equation (2) into Equation (1)

$$
2 i k \frac{\partial \tilde{E}}{\partial z}+\frac{\partial^{2} \tilde{E}}{\partial x^{2}}+\frac{\partial^{2} \tilde{E}}{\partial y^{2}}+k^{2} \tilde{\varepsilon}(\varrho, z) \tilde{E}=0
$$


According to the smooth perturbation method proposed by Rytov, the complex magnitude is written in the form of perturbation, thus,

$$
\tilde{E}(\varrho, z)=A_{0} \exp \left[i S^{\prime}(\varrho, z)+\ln \frac{A(\varrho, z)}{A_{0}}\right]
$$

where $S^{\prime}=S-k z$ is the offset of phase fluctuation caused by atmospheric turbulence, and

$$
S=k \varphi
$$

where $S$ is the phase, $\varphi$ is the optical path function. Let

$$
\chi=\ln \frac{A(\varrho, z)}{A_{0}}
$$

indicate the amplitude fluctuation, where $A(\varrho, z)$ is the random amplitude in atmospheric turbulence. The following formula can be obtained by substituting Equation (4) into Equation (3), thus

$$
2 i k \frac{\partial \phi}{\partial z}+\frac{\partial^{2} \phi}{\partial x^{2}}+\frac{\partial^{2} \phi}{\partial y^{2}}+\left(\frac{\partial \phi}{\partial x}\right)^{2}+\left(\frac{\partial \phi}{\partial y}\right)^{2}+k^{2} \tilde{\varepsilon}(\varrho, z)=0
$$

where $\phi=\chi+i S^{\prime}$ is the turbulence fluctuation. To study the relationship of point coordinates of optical propagation, the Green formula is introduced.

$$
K\left(\varrho-\varrho^{\prime}, z-z^{\prime}\right)=-\frac{1}{4 \pi\left(z-z^{\prime}\right)} \exp \left[\frac{i k\left(\varrho-\varrho^{\prime}\right)^{2}}{2\left(z-z^{\prime}\right)}\right]
$$

where $\varrho$ and $\varrho^{\prime}$ are abscissa of studied point and abscissa of reference point; $z$ and $z^{\prime}$ are ordinate of studied point and ordinate of reference point. The following formula can be obtained by solving Equation (7).

$$
\phi(\varrho, z)=-\int_{0}^{z} d z^{\prime} \int K\left(\varrho-\varrho^{\prime}, z-z^{\prime}\right) k^{2} \tilde{\varepsilon}\left(\varrho^{\prime}, z^{\prime}\right) d \varrho^{\prime}
$$

To further solve Equation (9), the Fourier transform transition quantities $\xi(\kappa, z)$ and $\eta(\kappa, z)$ are introduced, thus

$$
\begin{aligned}
\phi(\varrho, z) & =\int \xi(\kappa, z) \exp (i \boldsymbol{\kappa} \cdot \varrho) d \kappa \\
k^{2} \tilde{\varepsilon}(\varrho, z) & =-\int \eta(\kappa, z) \exp (i \boldsymbol{\kappa} \cdot \varrho) d \kappa
\end{aligned}
$$

where $k$ is the random wave number of turbulence medium. By combining with Equations (7), (9), (10) and (11), $\xi(\kappa, z)$ can be written as

$$
\xi(\kappa, z)=\frac{i k}{2} \int_{0}^{z} \exp \left[-\frac{i \kappa^{2}\left(z-z^{\prime}\right)}{2 k}\right] \varepsilon\left(\kappa, z^{\prime}\right) d z^{\prime}
$$

where $\varepsilon\left(\kappa, z^{\prime}\right)$ is the random dielectric constant under the atmospheric turbulence. The following formula can be obtained by substituting Equation (12) into (10).

$$
\phi(\varrho, z)=\frac{i k}{2} \int\left\{\int_{0}^{z} \exp \left[-\frac{i \kappa^{2}\left(z-z^{\prime}\right)}{2 k}\right] \varepsilon\left(\kappa, z^{\prime}\right) d z\right\} \exp (i \kappa \cdot \varrho) d \kappa
$$

The following formula can be obtained by solving conjugate for Equation 
(13).

$$
\phi^{*}(\varrho, z)=-\frac{i k}{2} \int\left\{\int_{0}^{z} \exp \left[\frac{i \kappa^{2}\left(z-z^{\prime}\right)}{2 k}\right] \varepsilon\left(\kappa, z^{\prime}\right) d z\right\} \exp (i \boldsymbol{\kappa} \cdot \varrho) d \kappa
$$

Thus, the following formula can be obtained.

$$
\chi(\varrho, z)=\int \chi(\kappa, z) \exp (i \boldsymbol{\kappa} \cdot \varrho) d \kappa=\int \frac{\varphi(\kappa, z)+\varphi^{*}(-\kappa, z)}{2} \exp (i \boldsymbol{\kappa} \cdot \varrho) d \kappa
$$

If using $\chi(\varrho, z)$ to indicate the frequency fluctuation of amplitude, thus

$$
\chi(\kappa, z)=\frac{\varphi(\kappa, z)+\varphi^{*}(-\kappa, z)}{2}
$$

By substituting Equation (12) and its conjugate into Equation (16), the following formula can be obtained.

$$
\chi(\kappa, z)=\frac{k}{2} \int_{0}^{z} \sin \left[\frac{\kappa^{2}\left(z-z^{\prime}\right)}{2 k}\right] \varepsilon\left(\kappa, z^{\prime}\right) d z^{\prime}
$$

If the covariance of fluctuation of dielectric constant in the medium satisfies the Gauss's law, the following formula is established.

$$
\Phi_{\varepsilon}(\kappa)=\frac{\sigma_{\varepsilon}^{2} a^{3}}{(2 \pi)^{\frac{3}{2}}} \exp \left(-\frac{\kappa^{2} a^{2}}{2}\right)
$$

where $\sigma_{\varepsilon}^{2}$ is the dielectric variance; and a is the radius of cross-section of propagating laser. The following formula can be obtained according to the covariance formula.

$$
\left\langle\chi(\kappa, z) \chi\left(\kappa^{\prime}, z\right)\right\rangle=\delta\left(\kappa+\kappa^{\prime}\right) F_{\chi}(\kappa, z)
$$

where $\delta\left(\kappa+\kappa^{\prime}\right)$ is the unit tensor of covariance; and $F_{\chi}(\kappa, z)$ is the two-dimensional spectrum density of amplitude fluctuation.

$$
\left\langle\chi(\kappa, z) \chi\left(\kappa^{\prime}, z\right)\right\rangle=\frac{k^{2}}{4} \iint_{0}^{z} \cos \frac{\kappa^{2}\left(z-z^{\prime}\right)}{2 k} \cos \frac{\kappa^{\prime 2}\left(z-z^{\prime \prime}\right)}{2 k} \varepsilon\left(\kappa, z^{\prime}\right) \varepsilon\left(\kappa^{\prime}, z^{\prime \prime}\right) d z^{\prime} d z^{\prime \prime}
$$

According to covariance relationship of fluctuation medium, the following formula can be obtained.

$$
\left\langle\varepsilon\left(\kappa, z^{\prime}\right) \varepsilon\left(\kappa^{\prime}, z^{\prime \prime}\right)\right\rangle=\delta\left(\kappa+\kappa^{\prime}\right) F_{\varepsilon}\left(\kappa, z^{\prime}-z^{\prime \prime}\right)
$$

Thus the spectrum density can be indicated as

$$
F_{\chi}(\kappa, z)=\frac{k^{2}}{4} \iint_{0}^{z} \cos \frac{\kappa^{2}\left(z-z^{\prime}\right)}{2 k} \cos \frac{\kappa^{2}\left(z-z^{\prime \prime}\right)}{2 k} F_{\varepsilon}\left(\kappa, z^{\prime}-z^{\prime \prime}\right) d z^{\prime} d z^{\prime \prime}
$$

By substituting the three-dimensional spectrum density of fluctuation medium, Equation (22) can be indicated as

$$
\begin{aligned}
F_{\chi}(\boldsymbol{\kappa}, z) & =\frac{\pi k^{2}}{2} \Phi_{\varepsilon}(\kappa, 0) \int_{0}^{z} \cos \frac{\kappa^{2}\left(z-z^{\prime}\right)}{2 k} d z^{\prime} \\
& =\frac{\pi k^{2} z \Phi_{\varepsilon}(\kappa, 0)}{4}\left(1+\frac{k}{\kappa^{2} z} \sin \frac{\kappa^{2} z}{k}\right)
\end{aligned}
$$


According to

$$
\psi_{\chi}(\varrho, z)=\int F_{\chi}(\kappa, z) \exp (i \kappa \cdot \varrho) d \kappa
$$

the covariance formula of amplitude fluctuation can be obtained as following.

$$
\psi_{\chi}(\varrho, z)=\frac{\pi^{2} k^{2} z}{2} \int_{z}^{\infty} J_{0}(\kappa \varrho) \Phi_{\varepsilon}(\kappa, 0) \kappa\left(1-\frac{k}{\kappa^{2} z} \sin \frac{\kappa^{2} z}{k}\right) d \kappa
$$

where $J_{0}(\kappa \varrho)$ is the zero-order Bessel function.

According to the principle of statistical optics [19], $\sigma_{\chi}^{2}=\psi_{\chi}(0, z)$ is established, thus the function of amplitude fluctuation can be written as following.

$$
\sigma_{\chi}^{2}=\frac{\pi^{2} k^{2} z}{2} \int_{0}^{\infty} \Phi_{\varepsilon}(\kappa, 0) \kappa\left(1-\frac{k}{\kappa^{2} z} \sin \frac{\kappa^{2} z}{k}\right) d \kappa
$$

The following formula can be obtained by substituting Equation (16) into Equation (26).

$$
\sigma_{\chi}^{2}=\frac{\sqrt{2 \pi} a^{3} k^{2} \sigma_{\varepsilon}^{2} z}{8} \int_{0}^{\infty} \exp \left(-\frac{a^{2} \kappa^{2}}{2}\right) \kappa\left(1-\frac{k}{\kappa^{2} z} \sin \frac{\kappa^{2} z}{k}\right) d \kappa
$$

To make the expression more intuitional, let $x=\frac{a^{2} \kappa^{2}}{2}$, thus Equation (27) can be written as

$$
\sigma_{\chi}^{2}=\frac{\sqrt{2 \pi} a k^{2} \sigma_{\varepsilon}^{2} z}{8} \int_{0}^{\infty}\left[1-\operatorname{sinc}\left(\frac{2 x z}{a^{2} k}\right)\right] e^{-x} d x
$$

According to the computational relationship, the following formula can be obtained

$$
\arctan \left(\frac{2 z}{a^{2} k}\right)=\int_{0}^{\frac{2 z}{a^{2} k}} \frac{1}{1+z^{\prime 2}} d z^{\prime}
$$

Thus, the function of amplitude fluctuation can be written in the final indication form as following.

$$
\sigma_{\chi}^{2}=\frac{\sqrt{2 \pi}}{16} a^{3} k^{3} \sigma_{\varepsilon}^{2}\left(\frac{2 z}{a^{2} k}-\arctan \frac{2 z}{a^{2} k}\right)
$$

\section{Result and Discussion}

It can be seen from Equation (30) that when the characteristic of laser and transmission distance are confirmed, the fluctuation variance of dielectric constant is proportional with amplitude fluctuation. The fluctuation degree of dielectric constant caused by the atmospheric turbulence medium is equal with the amplitude fluctuation. Let $\sigma_{\varepsilon}^{2}$ be the statistical unit quantity, thus the influences of other factors on amplitude can be easily confirmed. When the radius of cross-section of laser is $0.01 \mathrm{~m}$, and the transmission distance is $200 \mathrm{~m}, 500 \mathrm{~m}$ and $1000 \mathrm{~m}$ respectively, the relationships between wave number and amplitude fluctuation are shown in Figure 1.

It can be seen from the above figure that with the reduction of wave number, 
namely with the increase of wavelength, the fluctuation degree of amplitude reduces. With the reduction of wavelength, the fluctuation degree of amplitude increases, and the increasing slope grows bigger slowly. It can be seen that with the increase of selected wavelength, the amplitude fluctuation reduces, which is beneficial for propagation of laser. Moreover, the transmission distance also has significant influence on fluctuation degree of amplitude. In Figure 1, the slope of amplitude fluctuation increases most significant when the transmission distance is $1000 \mathrm{~m}$, indicating the transmission distance is an important factor of transmission of optical wave energy. Thus, the measurement range should be cleared in practice.

When the wavelength is $632.8 \mathrm{~nm}$, and the transmission distance is $200 \mathrm{~m}, 500$ $\mathrm{m}$ and $1,000 \mathrm{~m}$ respectively, the relationships between radius of cross-section of laser and amplitude fluctuation are shown in Figure 2.

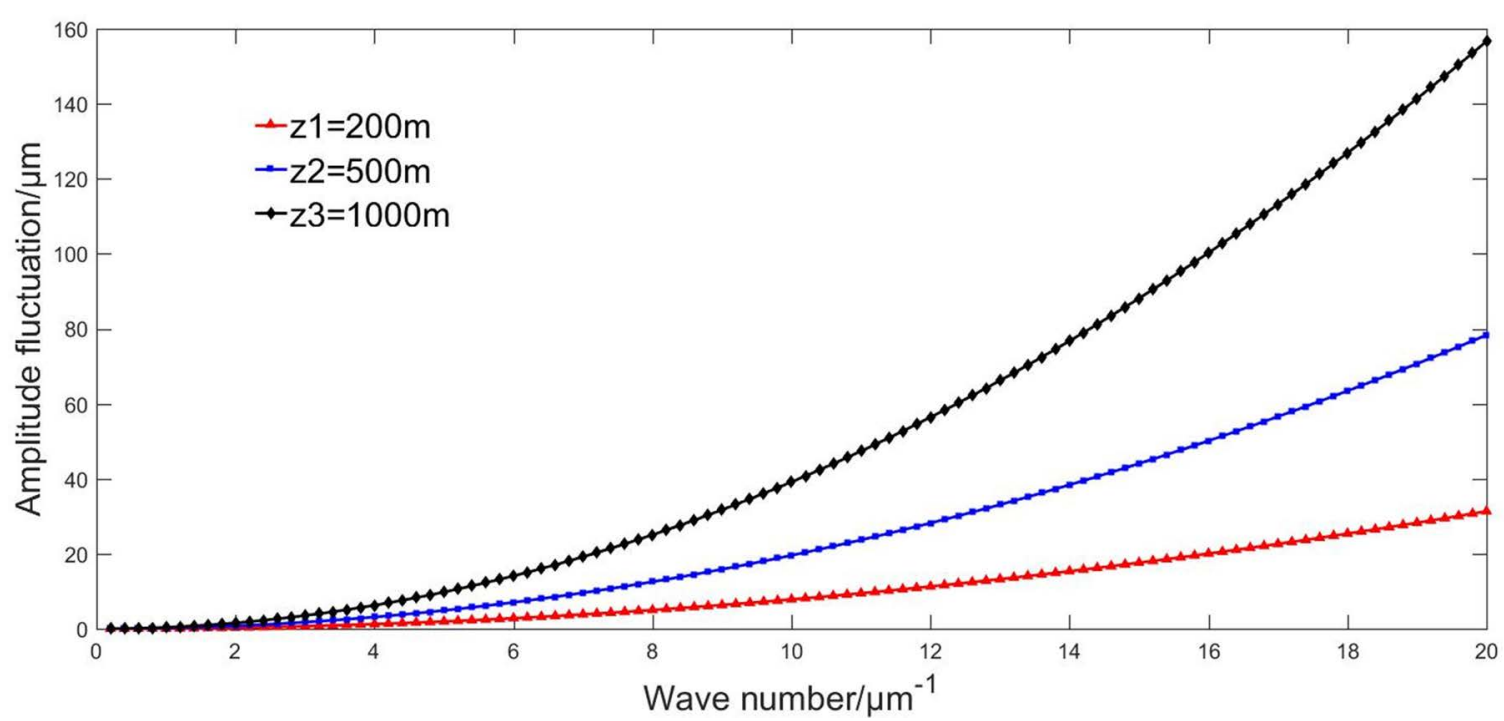

Figure 1. Relationship between wave number and amplitude fluctuation.

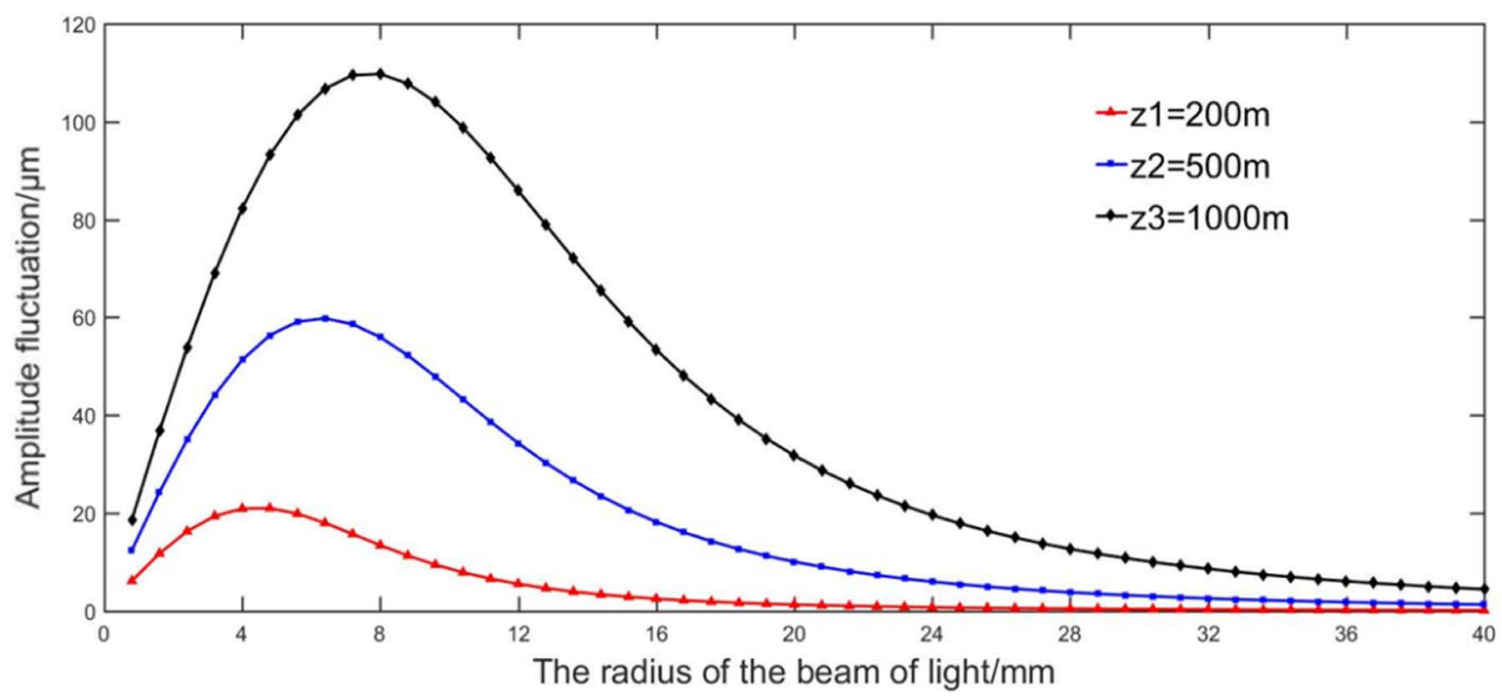

Figure 2. Relationship between radius of cross-section of laser and amplitude fluctuation. 
With the increase of radius of cross-section of laser, the amplitude fluctuation increases greatly and then reduces gradually. Until the radius of cross-section is very big, the amplitude fluctuation reaches to be stable and is close to be 0 . There is a peak of amplitude fluctuation when the radius of cross-section is between 4 $\mathrm{mm}$ and $10 \mathrm{~mm}$, indicating the atmospheric turbulence medium has a significant influence on transmission characteristic of cross-section in this range. This is not beneficial for receiving echo signal of optical information, and the interference compensation optical path is not easy to be realized. It can be seen from Figure 2 that with the increase of transmission distance, the amplitude fluctuation peak comes later, but the curves have the same stable trend finally. Therefore, the radius of cross-section of laser should increase as much as possible when satisfying related conditions. This not only can make the transmission flux sufficient, but also can make the amplitude fluctuation not obvious, so as to acquire effective laser.

When the wavelength is $632.8 \mathrm{~nm}$, and the radius of cross-section of laser is 5 $\mathrm{mm}, 10 \mathrm{~mm}$ and $40 \mathrm{~mm}$ respectively, the relationships between transmission distance and amplitude fluctuation are shown in Figure 3.

The fluctuation degree of amplitude increases with the increase of transmission distance. It can be obviously seen that when the radius of cross-section of laser is $5 \mathrm{~mm}$, the change of transmission distance has a significant influence on amplitude fluctuation. The fluctuation degree of amplitude reduces obviously with the increase of radius of cross-section. When the radius is $40 \mathrm{~mm}$, the amplitude fluctuation is very slight. However, with the increase of radius of crosssection of laser, the power is consumed more, and the carry and installation are more difficult. Thus, the parameters should be selected with comprehensive consideration. To observe the comprehensive influence of radius of cross-section of laser and transmission distance on amplitude fluctuation degree more intuitively, the wavelength is still selected as $632.8 \mathrm{~nm}$, as shown in Figure 4.

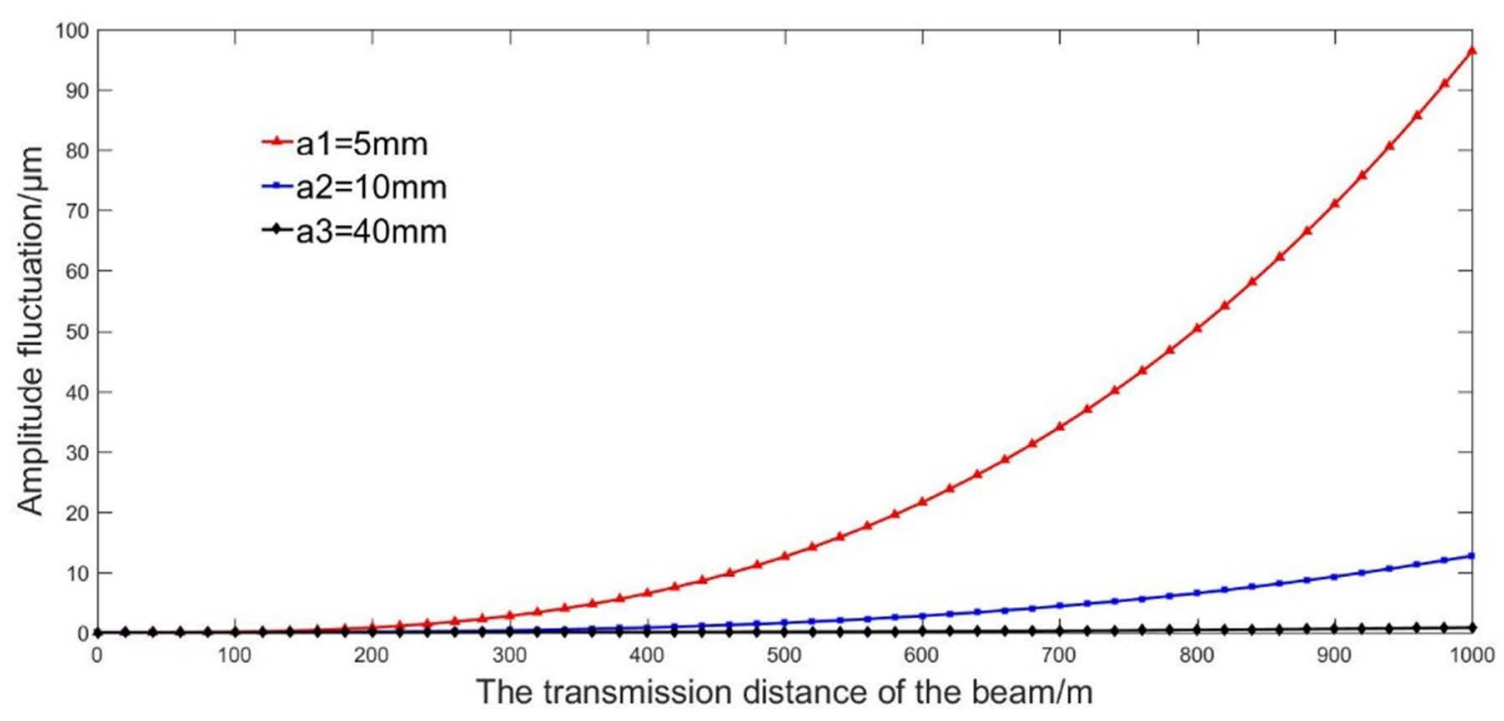

Figure 3. Relationship between transmission distance and amplitude fluctuation. 
Figure 4 reflects that the change of transmission distance of optical wave can generate serious fluctuation of amplitude, and its relative influencing degree is more obvious than that of radius of cross-section. In the process of near field transmission, the influences of radius of cross-section and transmission distance on amplitude fluctuation are relatively stable, which do not have big change. When the laser transmits and detects in long distance, the radius of cross-section should be selected appropriately, so as to reduce the amplitude fluctuation and to assure the stability of information reception.

When the laser transmits in the real atmosphere, the light intensity on crosssection has change as influenced by atmospheric turbulence, which can reflect the amplitude fluctuation. The test was conducted on a fine day. When the transmission distance of incident laser is $300 \mathrm{~m}$ and $1000 \mathrm{~m}$, respectively, the obtained light spots are shown in Figure 5 [20].

It can be seen from Figure 5 that the transmission distance of laser has a significant influence on amplitude fluctuation of light spot. During the transmission process, the light spot of initial laser is no longer regular, which has obvious speckle phenomenon. The influence of atmospheric turbulence on amplitude

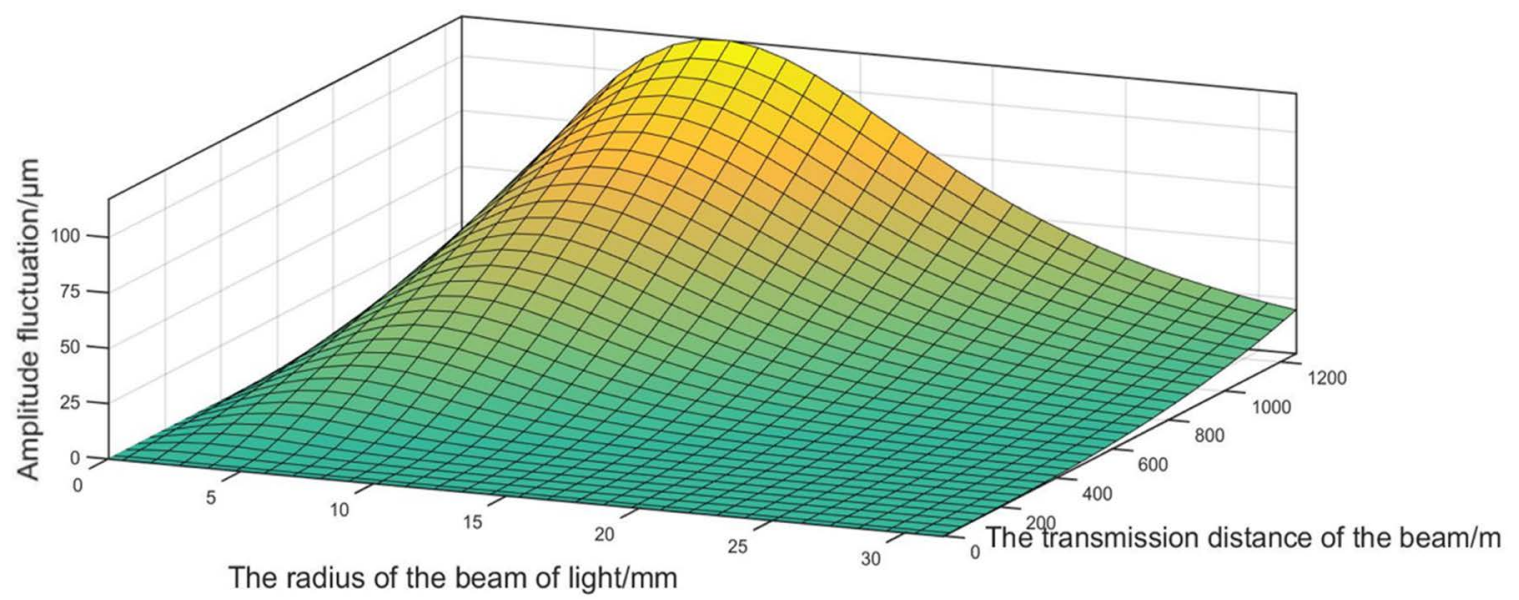

Figure 4. Intuitional diagram of comprehensive influencing factors of amplitude fluctuation degree.

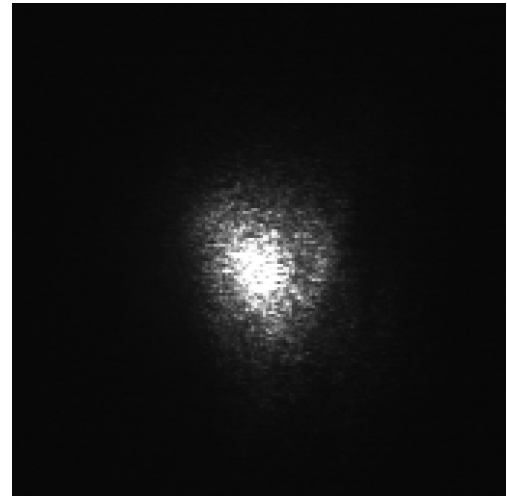

(a)

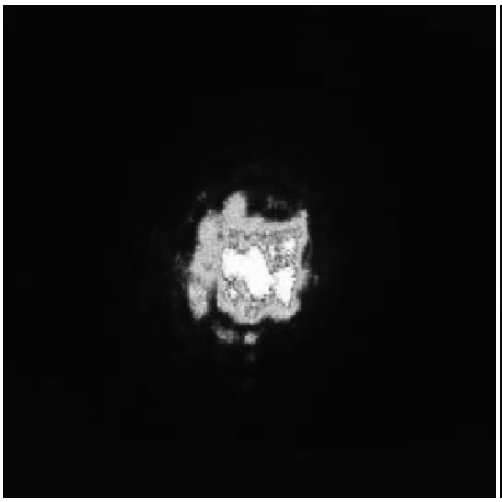

(b)

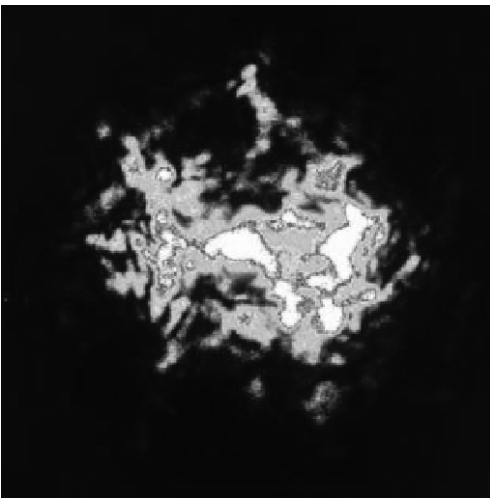

(c)

Figure 5. Actual light spots of laser. (a) Light sport of incident laser; (b) Light sport of laser at $300 \mathrm{~m}$; (c) Light sport of laser at $1000 \mathrm{~m}$. 
and light intensity is a key factor in actual measurement.

\section{Conclusions}

This paper studied the influences of optical wave attributes and transmission distance on amplitude fluctuation in turbulence medium. The study shows that the amplitude fluctuation reduces with the increase of wavelength. The amplitude fluctuation is even close to be ideal in the near-infrared field. With the increase of radius of cross-section of laser, the amplitude fluctuation has a peak then reduces slowly. The transmission distance has the most obvious influence on amplitude fluctuation. With the increase of transmission distance, the amplitude fluctuation grows more serious, but it can be adjusted by selecting appropriate radius of cross-section. It is an effective solution for assuring to acquire amplitude effectively to select the appropriate wavelength and radius of cross-section under the specific transmission distance.

In the practical transmission of laser, the energy loss increases with the increase of transmission distance as the atmosphere absorbs and scatters the energy of laser. Thus the amplitude acquired at last has more obvious fluctuation. Except adjusting the attributes of laser itself, the received information also can be improved by adjusting the phase parameters of reference optical path in the interference measurement field. We will study the parameters of reference optical path further in the subsequent work, so as to apply the transmission of laser in turbulence medium in the interference measurement and to provide theoretical basis for interference measurement technology in long distance.

\section{Conflicts of Interest}

The authors declare no conflicts of interest regarding the publication of this paper.

\section{References}

[1] Kaushal, H. and Kaddoum, G. (2017) Optical Communication in Space: Challenges and Mitigation Techniques. IEEE Communications Surveys \& Tutorials, PP, 1-1. https://doi.org/10.1109/COMST.2016.2603518

[2] Xu, E.-B., Guo, Y.-K., Liu, C., et al. (2017) Single Particle Scattering Light Detection Circuit with Low Noise. Semiconductor Optoelectronics, 38, 414-418.

[3] Ellis, D.D., Jie, Y., Preston, J.R., et al. (2017) A Normal Mode Reverberation and Target Echo Model to Interpret Towed Array Data in the Target and Reverberation Experiments. IEEE Journal of Oceanic Engineering, PP, 1-18.

[4] Wang, Y.-P., Hu, Y.-H., Lei, W.-H., et al. (2017) Aircraft Target Classification Method Based on Texture Feature of Laser Echo Time-Frequency Image. Acta Optica Sinica, 37, Article ID: 1128004. https://doi.org/10.3788/AOS201737.1128004

[5] Tang, M.-R., Li, Z.-L., Li, Y.-Q., et al. (2017) Influence of Atmospheric Turbulence Intermittency on Return Photon Number in Laser Ranging. Acta Optica Sinica, 37, Article ID: 0201001. https://doi.org/10.3788/AOS201737.0201001

[6] Surof, J., Poliak, J. and Calvo, R.M. (2017) Demonstration of Intradyne BPSK Opti- 
cal Free-Space Transmission in Representative Atmospheric Turbulence Conditions for Geostationary Uplink Channel. Optics Letters, 42, 2173.

https://doi.org/10.1364/OL.42.002173

[7] Rahman, A.K., Julai, N., Jusoh, M., et al. (2017) High Range Free Space Optic Transmission Using New Dual Diffuser Modulation Technique. European Physical Journal Web of Conferences, 12, 1026-1030.

[8] Venkatesh Murthy, B.T. and Srinivasa Rao, I. (2016) High-Gain Sub-Decibel Noise Figure Low Noise Amplifier for Atmospheric Radar. Microwave \& Optical Technology Letters, 58, 1618-1622. https://doi.org/10.1002/mop.29873

[9] Yu, L., Hu, Y.-H., Xu, S.-L., et al. (2016) Modeling and Simulation of Coherent Laser Detection of the Wind Field Disturbance. Journal of Optoelectronics Laser, 27, 973-979.

[10] Klimenko, V.V. and Mareev, E.A. (2018) Anomalous Decimeter Radio Noise from the Region of the Atmospheric Front: I. Characteristics of the Detected Radio Noise and Meteorological Parameters of the Frontal Cloudiness. Izvestiya Atmospheric \& Oceanic Physics, 54, 147-153. https://doi.org/10.1134/S0001433818020135

[11] Haque, M.I. and Basak, R. (2017) Land Cover Change Detection Using GIS and Remote Sensing Techniques: A Spatio-Temporal Study on Tanguar Haor, Sunamganj, Bangladesh. Egyptian Journal of Remote Sensing \& Space Science, 20, 251-263. https://doi.org/10.1016/j.ejrs.2016.12.003

[12] Afrasinei, G.M., Melis, M.T., Buttau, C., et al. (2017) Assessment of Remote Sensing-Based Classification Methods for Change Detection of Salt-Affected Areas. Journal of Applied Remote Sensing, 13, 151-155.

[13] Minati, L., Faes, L., Frasca, M., et al. (2018) Apparent Remote Synchronization of Amplitudes: A Demodulation and Interference Effect. Chaos, 28, 3124-3129. https://doi.org/10.1063/1.5026980

[14] Cordova, C., Maldacena, J. and Turiaci, G.J. (2017) Bounds on OPE Coefficients from Interference Effects in the Conformal Collider. Journal of High Energy Physics, 2017, 32. https://doi.org/10.1007/JHEP11(2017)032

[15] Abuturab, M.R. (2017) Multiple Color-Image Fusion and Watermarking Based on Optical Interference and Wavelet Transform. Optics \& Lasers in Engineering, 8, 47-58. https://doi.org/10.1016/j.optlaseng.2016.02.014

[16] Denis, D.C.B., Da Fonseca, A.F. and Mello, L.F. (2017) Melnikov Functions and Limit Cycles in Piecewise Smooth Perturbations of a Linear Center Using Regularization Method. Nonlinear Analysis Real World Applications, 36, 101-114. https://doi.org/10.1016/j.nonrwa.2017.01.003

[17] Rytov, S.M., Kravtsov, Y.A. and Tatarskii, V.I. (2018) Principles of Statistical Radiophysics. Springer-Verlag, New York.

[18] Beran, M.J. (19900 Book Review: Principles of Statistical Radiophysics, 4, Elements of Random Fields. Journal of Statistical Physics, 61, 957-958. https://doi.org/10.1007/BF01027316

[19] Joseph, W.G. (2017) Statistical Optics. CHEN Jia-Bi, Qin Ke-Cheng, Cao Qi-Zhi, transl. Science Press, Beijing.

[20] He, W. (2012) Simulation Study on Laser Propagation through the Atmosphere in Optoelectronic Countermeasures. University of Electronic Science and Technology of China. 